\title{
Enhanced recovery after surgery in liver surgery
}

\author{
Niteen Kumar, Sandeep K. Jha, Sanjay Singh Negi \\ Department of HPB Surgery and Liver Transplant, BLK Superspeciality Hospital, New Delhi 110005, India.
}

Correspondence to: Sanjay Singh Negi, Department of HPB Surgery and Liver Transplant, BLK Superspeciality Hospital, New Delhi 110005, India. Email: drsanjaynegi@gmail.com

How to cite this article: Kumar N, Jha SK, Negi SS. Enhanced recovery after surgery in liver surgery. Mini-invasive Surg 2018;2:41. http://dx.doi.org/10.20517/2574-1225.2018.49

Received: 8 Jul 2018 First Decision: 8 Oct 2018 Revised: 20 Nov 2018 Accepted: 22 Nov 2018 Published: 29 Nov 2018

Science Editor: Fernando Andrés Alvarez Copy Editor: Cui Yu Production Editor: Huan-Liang Wu

\begin{abstract}
Enhanced recovery after surgery (ERAS) or fast-track surgery protocols, have been implemented across surgical fields with positive impact on outcomes. These protocols represent a standardized and evidence-based multimodal perioperative strategy founded on a series of measures aiming to attenuate the physical and psychological stress responses to surgical insults, and to potentiate the postoperative rehabilitation of patients. The successful adoption of ERAS protocols in various specialties enabled its gradual acceptance in the complex field of liver surgery. Even though many elements have been adapted especially from colorectal surgery, a few elements of ERAS protocol are unique to liver surgery. The goals of enhanced recovery can be achieved with efforts beginning at the first interaction on outpatient basis. Core elements of this multidisciplinary effort include pre-operative counseling, shortened preoperative fasting, no pre-anesthetic medication, targeted antimicrobial prophylaxis and early withdrawal, preventing and treating of postoperative nausea and vomiting, minimally invasive approaches, avoidance of postoperative nasogastric decompression, preventing hypothermia, optimal perioperative fluid management, selective use of abdominal drains, early urinary catheter removal, optimal pain control, early oral feeding and mobilization. The available evidence from recent randomized controlled studies and meta-analyses comparing ERAS programs with traditional care in liver surgery suggests that length of hospital stay is shortened without increasing morbidity, mortality or readmission rates.
\end{abstract}

Keywords: Liver surgery, hepatic surgery, enhanced recovery after surgery, fast track, enhanced recovery

\section{INTRODUCTION}

Surgery alters the body physiology and defense mechanisms resulting in a catabolic state with impaired immunity, gut motility and respiratory physiology. These post-operative physiologic changes stem from metabolic, inflammatory or immunological responses and are thought to be primarily responsible for morbidity $^{[1]}$. Mechanistically, initiation of surgical stress response is primarily due to afferent nerve im-

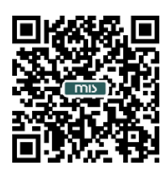


pulse combined with release of humoral substances [such as prostaglandins, kinins, leukotrienes, IL-1 and tumor necrosis factor (TNF)]. This phenomenon is amplified by factors including semi-starvation, infection and hemorrhage ${ }^{[2]}$. The neural pathway is probably most important in releasing the classic endocrine catabolic response, while associated release of humoral factors is important for the hyperthermic response, changes in coagulation and fibrinolysis, immune function and capillary permeability ${ }^{[1,2]}$. No wonder, attenuation of surgical stress response is dependent upon the technique of analgesia and afferent neural blockade with local anesthetic, with epidural being the most effective technique ${ }^{[1]}$. The introduction of effective neural blockade and use of epidural analgesia has been found to mitigate the metabolic response to surgical stress, but has a less important effect on inflammatory or immunologic responses. On the contrary, minimally invasive surgery reduces the inflammatory response and immune suppression, while leaving the metabolic response mostly unaltered ${ }^{[1]}$.

Even though the concept of bundling perioperative treatments to improve outcomes was developed in the early 90' and known as "Fast-Track" surgery, the enhanced recovery after surgery (ERAS) protocol was developed in Europe in $2001^{[3,4]}$. This last involved a more comprehensive multidisciplinary and multimodal approach with the main end-point of enhancing the quality of recovery by attenuating the perioperative surgical stress and improving the response to stress rather than just accelerating the speed of recovery ${ }^{[4]}$. Initially employed in colorectal surgery, it led to tremendous improvement in patient outcomes following surgery. This was later adopted by other surgical specialties including liver surgery. No wonder, many recommendations pertaining to ERAS in liver surgery arise from the experience in colorectal surgery ${ }^{[5]}$. However, there has been an increased need to develop liver specific programs to optimize ERAS protocols and outcome parameters given that features such as hepatic reserve evaluation, surgical complexity and risk of post-hepatectomy liver failure are unique to liver surgery. Naturally, many strategies employed to enhance recovery after liver surgery are similar to other surgical fields, including perioperative measures as well as postoperative recovery standardization [Table 1]. After Mackay and O'Dwyer ${ }^{[6]}$ reported their initial enhanced recovery protocols for liver resection, few ERAS protocols for liver surgery have been published, and data is limited mostly to observational studies and few randomized controlled trials (RCT) ${ }^{[7-9]}$. In addition, there is scarce data comparing ERAS programs with conventional liver surgery protocols, and meta-analyses including RCTs suffer from inadequate disclosure of randomization techniques ${ }^{[9]}$. The subjective nature of end points (i.e., time to flatus) and the heterogeneity in outcome measures between studies lead to inherent imprecise data [i.e., length of stay (LOS), where the patient may be fit for discharge but stay in hospital due to logistic reasons]. Primary surgeon experience, baseline patient characteristics and selection criteria may also significantly impact the validity of results. Hence, even though there is a growing body of evidence in favor of ERAS application in liver surgery, further studies are required to determine the most effective ERAS protocol for this particular field. The purpose of this review is to summarize the current scientific evidence on the most important elements of an ERAS program in liver surgery and the outcomes associated with the application of this protocol compared to traditional care.

\section{KEY COMPONENTS OF ERAS PROTOCOLS IN LIVER SURGERY}

Implementation of the complete set of traditional core elements of ERAS protocols is rarely seen in referral liver surgery centers ${ }^{[10]}$. Although pre- and perioperative elements have a good adherence, compliance is especially poor for the postoperative phase elements ${ }^{[10]}$. This may be due to the fact that at least 7 out of 23 classical ERAS items validated for colorectal surgery have not been studied in liver surgery yet, and it is currently unclear whether they can be extrapolated for liver surgery ${ }^{[5]}$. However, some have been considered of outmost importance for liver surgery in recent meta-analyses and a consensus guideline from the international ERAS Society ${ }^{[5,9]}$. Specifically, pre- and intraoperative fluid restriction and no routine nasogastric tubes (NGTs) postoperatively have been considered significant for liver surgery ${ }^{[9]}$. On the contrary, elements such as preoperative oral mechanical bowel preparation and postoperative stimulation of bowel movement are not considered indicated in liver surgery ${ }^{[5]}$. 
Table 1. Core elements for enhanced recovery after surgery protocols in liver resections

\begin{tabular}{|c|c|c|c|}
\hline Pre-operative & Peri-operative & Post-operative & At discharge \\
\hline $\begin{array}{l}\text { - Education, counselling and } \\
\text { exercise } \\
\text { - No bowel preparation } \\
\text { - No preanesthetic medications } \\
\text { - Carbohydrate loading } 2 \text { h prior } \\
\text { surgery } \\
\text { - Minimal fasting ( } 2 \text { h) }\end{array}$ & $\begin{array}{l}\text { - Antibiotic prophylaxis } \\
\text { - Thromboembolic prophylaxis } \\
\text { - Epidural analgesia } \\
\text { - Short-acting i.v. anesthetic agent } \\
\text { - Prevention of hypothermia } \\
\text { - Optimal fluid balance no abdomi- } \\
\text { - } \text { nal drains or early removal } \\
\text { - Minimal incisions }\end{array}$ & $\begin{array}{l}\text { - No nasogastric tube } \\
\text { - Selective ICU transfer } \\
\text { - Multimodal analgesia } \\
\text { - Early removal of Foley's catheter } \\
\text { - Early enteral intake } \\
\text { - Early ambulation } \\
\text { - Early withdraw of i.v. fluids } \\
\text { - Early restoration of normal diet } \\
\text { - Glucose Control } \\
\quad \text { PONV prophylaxis }\end{array}$ & $\begin{array}{l}\text { - Normal or decreasing serum } \\
\text { bilirubin } \\
\text { - Good pain control with oral } \\
\text { analgesia only } \\
\text { - Tolerance of solid food } \\
\text { - No i.v. fluids } \\
\text { - Mobile independently or at } \\
\text { - } \text { the preoperative level } \\
\text { - Normallingness to go home } \\
\text { - No incision infection }\end{array}$ \\
\hline
\end{tabular}

ICU: intensive care unit; PONV: postoperative nausea and vomiting

\section{Preoperative information and counselling}

Fear and stress are common prior to surgery. Hospitalization is a stressful event that disturbs the physical and psychological wellbeing of a patient. Stress due to apprehension of surgery leads to activation of sympathetic axis and adrenaline overdrive. Increased cortisol and catecholamine production can significantly impact the healing process and particularly the initial inflammatory phase ${ }^{[11]}$. Although no high level evidence exists certifying the beneficial impact of preoperative counseling and education on outcomes, there is no doubt that education aids such as brochures, leaflets and online information help the patients in decision making and enhance the validity of informed consent $t^{[5]}$. Also, the engagement of visual media regarding the recovery process and postoperative expectations improves overall compliance with feeding and physiotherapy, hence reducing morbidity ${ }^{[12]}$. Whatever approach is employed, detailed information about the natural history of disease, surgical procedure, anesthesia, expected course of recovery and complications reduces stress and anxiety related to the procedure, which positively impacts postoperative outcomes. Earlier return of gastrointestinal (GI) motility has been shown in patients who received preoperative instruction compared to those who merely received information and reassurances ${ }^{[13]}$. Therefore, adequate counseling and communication with empathy may be all that is required sometimes to relieve postoperative ileus during the post-operative period ${ }^{[14]}$. Although there are no specific studies evaluating the therapeutic effect of preoperative counseling and patient education before liver surgery, it is strongly recommended for any ERAS protocol to include thorough preoperative information and counseling in order to allay patients' fear and reduce psychological stress.

\section{Preoperative fasting}

The concept of overnight fasting before surgery to ensure an empty stomach and avoid pulmonary complications has been decisively challenged in recent years. Prolonged fasting leads to perioperative insulin resistance, fever, symptoms like malaise, hunger, thirst, nausea and increases patients' anxiety ${ }^{[15]}$. Fasting guidelines before surgery are based on gastric physiology and expert opinion, as there is limited evidence that they improve outcomes. Clear liquids and gastric secretion move rapidly out of the stomach, and even though glucose containing fluids leave the stomach more slowly, after $90 \mathrm{~min}$ the stomach is empty of clear liquids regardless of type ${ }^{[16]}$. Gastric residual volume averages about $25 \mathrm{~mL}$ in patients fasted overnight prior to surgery, and clear liquids intake up to $2 \mathrm{~h}$ before surgery does not seem to affect this residual volume ${ }^{[17,18]}$. In a Cochrane database systematic review, Brady et al. ${ }^{[19]}$ have shown that a liberal fluid fasting protocol does not increase the risk of aspiration or morbidity as compared to a conventional mid night fasting policy. Surgical insult following overnight fasting results in an exaggerated catabolic response that causes insulin resistance and prolongs recovery ${ }^{[2]}$. In fact, insulin resistance is a central metabolic change during surgical stress that is directly proportional to the magnitude of the operation and leads to hyperglycemia in non-diabetic patients ${ }^{[2]}$. As a consequence, various endocrine and inflammatory systems are stimulated. This results in an exacerbation of the existing postoperative catabolic state with marked loss 
of body fat and protein stores ${ }^{[20]}$. Given that postoperative insulin resistance has been associated with poor pain control, increased morbidity, and increased length of hospital stay after surgery, several studies have examined the impact of preoperative carbohydrate drink on patient well-being ${ }^{[2]}$. A review of 17 RCTs including 1445 patients concluded that patients receiving carbohydrates have reduced LOS, less perioperative insulin resistance and experience less fever, hunger, thirst, nausea and anxiety ${ }^{[21]}$. Hausel et al. ${ }^{[22]}$ investigated the impact of carbohydrate loading on postoperative nausea and vomiting (PONV) in 172 patients undergoing elective laparoscopic cholecystectomy. Within the first $24 \mathrm{~h}$ after surgery, the carbohydrate fed group experienced significantly lower incidence of PONV. Yet, other studies have not reported such beneficial effects ${ }^{[23]}$. Mathur et al. ${ }^{[24]}$ conducted a double-blind placebo RCT in 2009 to study the effect of preoperative carbohydrate drink on clinical outcomes after colorectal surgery and liver resection. The study however, did not observe any beneficial effects in LOS, oral intake or postoperative infections. Despite some controversy, carbohydrate loading has a solid physiologic foundation which is supported by several studies $^{[5]}$. Thus, incorporation of such strategies helps in patient's enhanced recovery, more so in the light of the fact that insulin resistance affects liver regeneration and might therefore have the potential of further delaying the recovery ${ }^{[2]}$. Overall, although preoperative fasting does not need to exceed $6 \mathrm{~h}$ for solids, a judicious recommendation for patients being operated at first time on the morning is to allow a normal meal the night before and continue carbohydrate liquids up to $2 \mathrm{~h}$ before induction of anesthesia.

\section{Antimicrobial prophylaxis}

Surgical site infection (SSI) is the most common postoperative complication after high-risk surgery, which increases both hospital stay and treatment costs ${ }^{[25]}$. Incidence of SSI after liver resection ranges between $2 \%$ and $15 \%$, and has significant impact on LOS, morbidity and mortality ${ }^{[26,27]}$. Even though liver surgery is considered a clean contaminated surgery, it is categorized as contaminated surgery when combined with extrahepatic bile duct resection due to the possibility of biliary or enteric spillage during surgery. In such scenarios, bile is almost always (up to $75 \%$ ) contaminated due to preoperative biliary drainage ${ }^{[28]}$. In addition, invasive nature of major hepatic resection and inevitable surgical field contamination has lead to the empirical use of prolonged antibiotic prophylaxis among surgeons. This practice, however, ends up increasing bacterial resistance and prolongs patient recovery. To date, three RCTs on postoperative antibiotic prophylaxis in patients undergoing hepatectomy without extrahepatic bile duct resection have been reported. Although only one of these RCTs has supported prolonged prophylaxis based on significant difference in the incidence of signs of infections or systemic inflammatory response syndrome, the incidence of infection did not differ among all RCTs ${ }^{[29-31]}$. In a more recent RCT by Sugawara et al. ${ }^{[32]}$, it was found that twoday administration of antibiotic prophylaxis is sufficient for patients undergoing complicated major hepatectomies with extrahepatic bile duct resection. Various other methods to reduce infectious complications have been attempted, including the use of pre- and pro-biotics, with no strong evidence of their efficacy ${ }^{[33]}$. In summary, it is recommended to administer a single dose of intravenous antibiotics before skin incision, of the type recommended by a local infectious committee. Postoperative "prophylactic" antibiotics are not recommended, with the exception of simultaneous bile duct resection in patients with a biliary drainage, where prophylaxis should be selected on the basis of preoperative surveillance bile cultures and discontinued on postoperative day (POD) 3, unless evidence to the contrary is shown.

\section{Antithrombotic prophylaxis}

Despite the common belief that liver resection impairs coagulation, increasing the risk of bleeding and protecting patient from thromboembolism, hypocoagulability is rare after liver resections ${ }^{[34]}$. A study done in living donor hepatectomy showed that pulmonary embolism is not rare despite prophylaxis with low molecular weight heparin $(\mathrm{LMWH})^{[35]}$. These clinical findings have been supported by thromboelastogram monitoring which shows a hypercoagulable state after liver resection due to imbalance in coagulation proteins ${ }^{[36,37]}$. Furthermore, major hepatectomy has been identified as an independent risk factor for pulmonary embolism ${ }^{[38]}$. A cohort study of 419 patients showed lower symptomatic postoperative venous thromboembolism if prophylaxis is initiated from day 1 after major hepatectomy ${ }^{[39]}$. One meta-analysis 
suggested continuing thromboprophylaxis for 4 weeks post-operatively, especially in patients bearing liver malignancies ${ }^{[40]}$. The combination of pharmacologic and mechanical prophylaxis, such as compressive stocking and intermittent pneumatic compression, may further reduce the risk in the high-risk group of patients $^{[41]}$. In summary, routine prophylaxis with LMWH or unfragmented heparin should be initiated 2-12 $\mathrm{h}$ before surgery in major hepatectomies, restarted $8-12 \mathrm{~h}$ after surgery if there are no signs of bleeding, and discontinued once the patient is discharged ${ }^{[5]}$. Given the absence of high-level evidence, extended thromboprophylaxis (28 days) should be considered only in selected patients with high-risk scores.

\section{Minimally invasive approach}

Even though none of the four incisions used for open liver surgery (median, Chevron, Mercedes-benz and Makuuchi) has shown to offer any advantages over the others and perioperative complications remain comparable, mini-invasive approaches have consistently demonstrated a substantial benefit with regards to patient recovery over the open approach. The central concept of surgical stress response attenuation orbits around the minimally invasive approach. Laparoscopic liver resection (LLR) was introduced in the

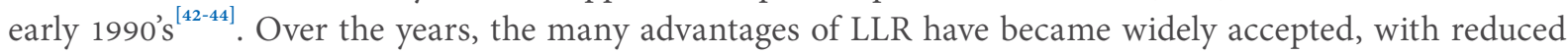
intraoperative bleeding, shorter LOS, less pain, lower infection rates, earlier recovery and better quality of life (QOL) ${ }^{[45-51]}$. In addition, emerging data has now confirmed the safety and oncologic equivalence of the laparoscopic approach for both malignant liver lesions ${ }^{[48,52-54]}$. A recent meta-analysis by Liu et al ${ }^{[55]}$ found that laparoscopic left lateral sectionectomy had significantly better results regarding blood transfusion, blood loss, total morbidity and LOS compared to the open approach. In fact, while major LLRs are still under development, minor LLRs including left lateral sectionectomy have become standard practice nowadays ${ }^{[47,48,55]}$. With regards to ERAS protocols in LLR, Stoot et al. ${ }^{[56]}$ reported from retrospective data a reduction in LOS from 7 days to 5 days when laparoscopy and ERAS program were combined. More recently, a propensity score-based analysis between the open and laparoscopic approaches from Ratti et al ${ }^{[57]}$ has found that the combination of a minimally invasive approach with a fast-track protocol allows a reduced rate of postoperative morbidity and satisfactory functional recovery, even in the setting of complex liver resections. Although laparoscopic surgery offers an additional advantage to ERAS protocols during postoperative recovery, adequate patient selection and surgeon expertise are key determinants of success ${ }^{[47,48]}$. Patients with lesions located in peripheral liver segments (Segments 2 to 6) that require minor resections ( $\leq$ segments) are considered the best candidates for this approach ${ }^{[47,48]}$. So far there are no studies assessing robotic liver surgery within ERAS frameworks.

\section{Prophylactic nasogastric intubation}

Pathogenesis of postoperative ileus as demonstrated by Wangensteen ${ }^{[58]}$ arose from excess of swallowed air, which can be relieved by NGT insertion. However, a NGT has been consistently associated with higher pulmonary complications and this may be due to several reasons ${ }^{[59]}$. First, it may be due to the incomplete closure of the glottis during cough hence leading to the accumulation of secretions, with increased risk of atelectasis and infection. Secondly, it acts as a conduit for transfer of bacteria from the oropharynx to the lungs. Thirdly, NGT also may cause diaphragmatic dysfunction through reflex mechanisms ${ }^{[60,61]}$. Time to passage of flatus and return to oral intake are delayed due to NGT, and around 70\% of patients experience marked discomfort limiting mobility with increased nursing care ${ }^{[62-64]}$. Furthermore, NGT is also associated with laryngeal injury, esophagitis, pharyngitis, otitis, electrolyte losses, aerophagia and rhinosinus$i^{i t i s}{ }^{[65-67]}$. A Cochrane review concluded that routine prophylactic use of NGT in general abdominal surgery can increase pulmonary complications and delay bowel function, therefore recommending its selective use $^{[68]}$. With regards specifically to NGT use after liver resections, two recent RCTs have confirmed the increased risk of complications and the absence of any advantages after elective liver surgery ${ }^{[59,69]}$. In summary, even though NGT decompression may be necessary during surgery, immediate on-table removal after surgery is strongly recommended as it has been proven to be safe and associated with better outcomes and an improved peri-operative experience for the patient ${ }^{[5]}$. 


\section{Prophylactic drainage}

An intense debate about the value and risk of prophylactic drainage in liver resections was raised in 2004 after a meta-analysis provided a strong ground to omit routine prophylactic drainage after major abdominal surgery ${ }^{[70]}$. Recent data from RCTs and large retrospective studies suggest that there is no evidence to support routine drain use after uncomplicated liver resections without bilio-enteric anastomosis ${ }^{[71,72]}$. Furthermore a routine use policy may even lead to an increased risk of complications and 30-day readmissions in major hepatectomy ${ }^{[73-75]}$. Overall, prophylactic drainage tubes should be used selectively and early removal is recommended in the absence of complications in order to promote easier mobilization.

\section{Postoperative mobilization and urinary catheter removal}

Bed rest in critically ill patients or after surgery can lead to muscular atrophy, weakness, joint contracture, thromboembolism, insulin resistance, microvascular dysfunction, systemic inflammation, atelectasis and bed sores ${ }^{[76]}$. Early physical activity during recovery from surgery has beneficial effect on many aspects of physiological functions. Up to $85 \%$ of patients undergoing liver resection may be ambulatory by postoperative day $3^{[7]}$. In a study by Yip et al ${ }^{[77]}$, sitting out of bed by POD $1(P<0.03)$, walking by POD $3(P=$ $0.03)$ and removal of urinary catheter by POD $3(P<0.01)$ were independently associated with successful completion of an ERAS protocol aiming at hospital discharge within 6 days after surgery. Delay in removal of urinary catheter is enough to prolong hospital stay. In a RCT, Zaouter et al. ${ }^{[78]}$ demonstrated catheter removal on POD 1 even with epidural analgesia had lower urinary infection rate and similar re-catheterization rates. A recent RCT by Ni et al. ${ }^{[79]}$ including 120 patients has shown that patients undergoing liver resection who perform early postoperative ambulation have statistically significant faster return of bowel function and shorter LOS, without increased risk of complications. Hence, early ambulation could reduce economic burden and nursing workload as well as increase patient comfort and satisfaction. In summary, early "out of bed" mobilization with daily goals adjusted to each individual should start the day after liver resection, as it is both feasible and safe, and it leads to faster patient recovery.

\section{Postoperative nutrition and early oral intake}

Allowing patients orally early after major upper GI surgery does not increase morbidity. A RCT on 427 patients, 66 of which had undergone hepatic resection or hepaticojejunostomy, confirmed the advantages and safety of normal oral nutrition at will from postoperative day ${ }^{[80]}$. Use of laxatives resulted in earlier passage of stools but the overall rate of recovery was unaltered ${ }^{[81]}$. Parenteral nutrition should be only used in mal-nourished patients or patients expected to have a prolonged fasting ( $>5$ days) and longer recovery due to complications or otherwise $e^{[5]}$. In summary, it is nowadays recommended that patients under an ERAS protocol should be allowed liquids the morning after surgery and switched to normal food by the evening if there is a good tolerance there are no complications ${ }^{[5]}$.

\section{Postoperative glycemic control}

Postoperative rise in blood glucose is expected due to deranged physiologic status of the body after major surgery. During hepatectomy, blood glucose levels shoot up sharply after Pringle maneouvre due to augmentation of glycogenolysis as a result of hypoxia ${ }^{[82]}$. In line with this concept, Hanazaki et al ${ }^{[83]}$ suggested that ischemic preconditioning may reduce the hyperglycemia caused by disturbances of hepatic glucose mechanism in association with ischemic reperfusion injury. Preoperative fasting combined with surgical stress response reduces liver glycogen stores and promotes insulin resistance with hyperglycemia ${ }^{[2]}$. Hyperglycemia is both a marker and cause of adverse outcomes both for diabetics and non-diabetic patients. The Interleukins released also cause insulin resistance either by suppressing insulin receptors tyrosine kinase activity or reduction of transmembrane glucose transporters expression, leading to hyperglycemia during early postoperative period ${ }^{[84]}$. It is therefore recommended to initiate insulin therapy early after liver resections in order to maintain normoglycemia ${ }^{[5]}$. 


\section{PONV}

Prevention and treatment of PONV is of utmost importance to allow early oral intake and keep the patient within an ERAS pathway. Risk factors include previous PONV, young female patient, nonsmoker, use of volatile anesthetic or opioids ${ }^{[5]}$. Given their favorable side effect profile, 5-HT3 antagonists such as Ondansetron, remain the treatment of choice ${ }^{[85]}$. Low dose dexamethasone has equivalent antiemetic action but has to be used with caution in diabetics ${ }^{[85]}$. Metoclopramide is a weak antiemetic and a dose of $10 \mathrm{mg}$ may not effectively reduce PONV ${ }^{[86]}$. Therefore, the international ERAS society and the international consensus group on PONV recommend a multimodal prophylaxis including at least two antiemetic drugs to reduce $\mathrm{PONV}^{[5,87]}$.

\section{Perioperative steroid administration}

Preoperative steroid in patients undergoing hepatic surgery is controversial and its use is limited. Although supported by experimental studies ${ }^{[8,89]}$, beneficial effects stemming from its immunologic and antiinflammatory action has not been consistent ${ }^{[90,91]}$. Although pre-operative steroid administration has not been associated with a reduction of post-operative complications in two recent meta-analysis of RCTs ${ }^{[00,91]}$, it resulted in significantly lower levels of serum bilirubin and interleukins on POD $1^{[92]}$. The mechanism of action may be due to a protection against warm ischemia-reperfusion injury, lower IL's release, better tissue perfusion, stabilization of cell membrane and lower lysosomal protease releas ${ }^{[93]}$. A negative effect of steroids in liver regeneration remains a concern, as IL- 6 and TNF- $\alpha$ are important initiators of hepatic regeneration ${ }^{[94]}$. However, Glanemann et al ${ }^{[95]}$ showed in an animal model that steroids had no negative impact on liver regeneration. Although the use of preoperative steroids (methylprednisolone) can not be strongly recommended in liver surgery, they may be used only before hepatectomy in non-diabetic patients with normal liver parenchyma in order to decrease liver injury and intraoperative stress.

\section{OUTCOMES OF ERAS PROTOCOLS COMPARED WITH TRADITIONAL CARE}

In the inaugural experience with a multimodal ERAS program after open liver surgery, Van Dam et al. ${ }^{[7]}$ reported a significant reduction in the LOS without increasing morbidity or mortality. Many later retrospective studies and meta-analyses comparing ERAS with traditional care have confirmed the safety and feasibility of ERAS in liver resection ${ }^{[96-98]}$. A recent meta-analysis by Wang et al. ${ }^{[9]}$ showed that hospital stay was significantly shorter for ERAS patients in both RCTs and non-RCTs, being reduced by a mean of 2.65 days and 1.81 days, respectively $(P<0.001)$. This benefit was increased if laparoscopic surgery was applied, with a mean reduction of 3.64 days $(P<0.001)^{[9]}$. Time to bowel function recovery has been consistently found significantly shorter when an ERAS protocol is applied ${ }^{[9,99]}$. With regards to morbidity, a meta-analysis of 4 RCTs found that complications were significantly reduced in ERAS patients compared to traditional care patients $(20.9 \% \text { vs. } 31.4 \% ; P=0.02)^{[99]}$. This was later confirmed by Wang et al. ${ }^{[9]}$, who found significantly less overall morbidity in both RCTs and non-RCTs $(\mathrm{OR}=0.57$ and 0.66 respectively; $P$ $=0.01$ ). However, when categorized according to the Dindo-Clavien classification, although ERAS group had significantly fewer grade I complications $(\mathrm{RR}=0.51 ; P=0.003)$, there were no differences in grade II-V complications $(\mathrm{RR}=0.94 ; P=0.80)^{[100]}$. Similarly, a RCT by Jones et al ${ }^{[97]}$ found a significantly reduced rate of medical complications ( $7 \%$ vs. $27 \% ; P=0.02)$, but not surgical complications $(15 \% v s .11 \% ; P=0.612)$. In addition, three meta-analyses of RCTs found no significant differences regarding 30-day mortality and readmission rates between ERAS and traditional care approaches ${ }^{[9,101,102]}$. With regards to QOL evaluation, two RCTs have found a statistically significant improvement in QOL by one month after surgery in ERAS patients ${ }^{[98,103]}$. Finally, although the benefits in outcomes of ERAS protocols have been translated in significant cost saving in colorectal surgery, from around $\$ 2,800$ to $\$ 5,900$ per patient, this has not been widely confirmed in liver surgery yet ${ }^{[4]}$. Although a recent retrospective cost-benefit analysis of ERAS in liver surgery from Switzerland found a total mean cost reduction of $€ 3,080$ per patient compared to traditional care, this difference did not reach statistical significance $(P=0.467)^{[104]}$. The main outcomes of ERAS protocols reported in the literature are summarized in Table 2. 
Table 2. Outcomes of enhanced recovery after surgery protocols in liver resections

\begin{tabular}{|c|c|c|c|c|c|c|c|}
\hline Ref. & Year & Study design & $n$ & $\begin{array}{l}\text { Length of stay, days } \\
\text { (Range) }\end{array}$ & Morbidity & Mortality & Results compared to TC \\
\hline Spelt et al. ${ }^{[96]}$ & 2011 & Review & 130 & $5-7$ & $15 \%-46 \%$ & $0 \%-1.8 \%$ & Reduced LOS \\
\hline Jones et $a{ }^{[97]}$ & 2013 & RCT & 46 & 4 & $7 \%$ & $2 \%$ & $\begin{array}{l}\text { Reduced LOS and morbidity } \\
\text { Improved QOL }\end{array}$ \\
\hline Ni et al. ${ }^{[98]}$ & 2013 & $\mathrm{RCT}$ & 80 & 5.2 & $30 \%$ & 0 & $\begin{array}{l}\text { Reduced morbidity, PONV, ileus and } \\
\text { LOS } \\
\text { Lower CRP }\end{array}$ \\
\hline Lei et $a .^{[99]}$ & 2014 & Meta-analysis of RCT's & 187 & $4-9.2$ & $20.9 \%$ & 0 & Reduced time to flatus, morbidity, LOS \\
\hline He et $a l^{[103]}$ & 2015 & $\mathrm{RCT}$ & 48 & $4-8$ & $14.6 \%$ & 0 & $\begin{array}{l}\text { Reduced LOS, time to flatus and cost } \\
\text { Improved QOL }\end{array}$ \\
\hline Ni et $a l^{[100]}$ & 2015 & Meta-analysis of RCT's & 354 & 5 & $15.5 \%$ & 0 & Reduced morbidity, ileus and LOS \\
\hline Liang et al. ${ }^{[105]}$ & 2016 & $\mathrm{RCT}$ & 80 & 6.2 & $22.5 \%$ & 0 & Reduced LOS, morbidity and cost \\
\hline Li et $a .^{[101]}$ & 2016 & Meta -analysis & 477 & $6-10$ & $29.7 \%$ & $0.02 \%$ & Reduced LOS \\
\hline Wang et $a{ }^{[9]}$ & 2017 & Meta-analysis & 1297 & $2.5-10$ & $28.4 \%$ & $0.49 \%$ & $\begin{array}{l}\text { Reduced morbidity, LOS, cost and } \\
\text { blood loss }\end{array}$ \\
\hline Rouxel et al. ${ }^{[102]}$ & 2018 & Review & 254 & $4-6.9$ & $7 \%-24 \%$ & $0.004 \%$ & Reduced morbidity and LOS \\
\hline
\end{tabular}

RCT: randomized controlled trial; TC: traditional care; LOS: length of hospital stay; QOL: quality of life; PONV: postoperative nausea and vomiting; CRP: $\mathrm{C}$-reactive protein

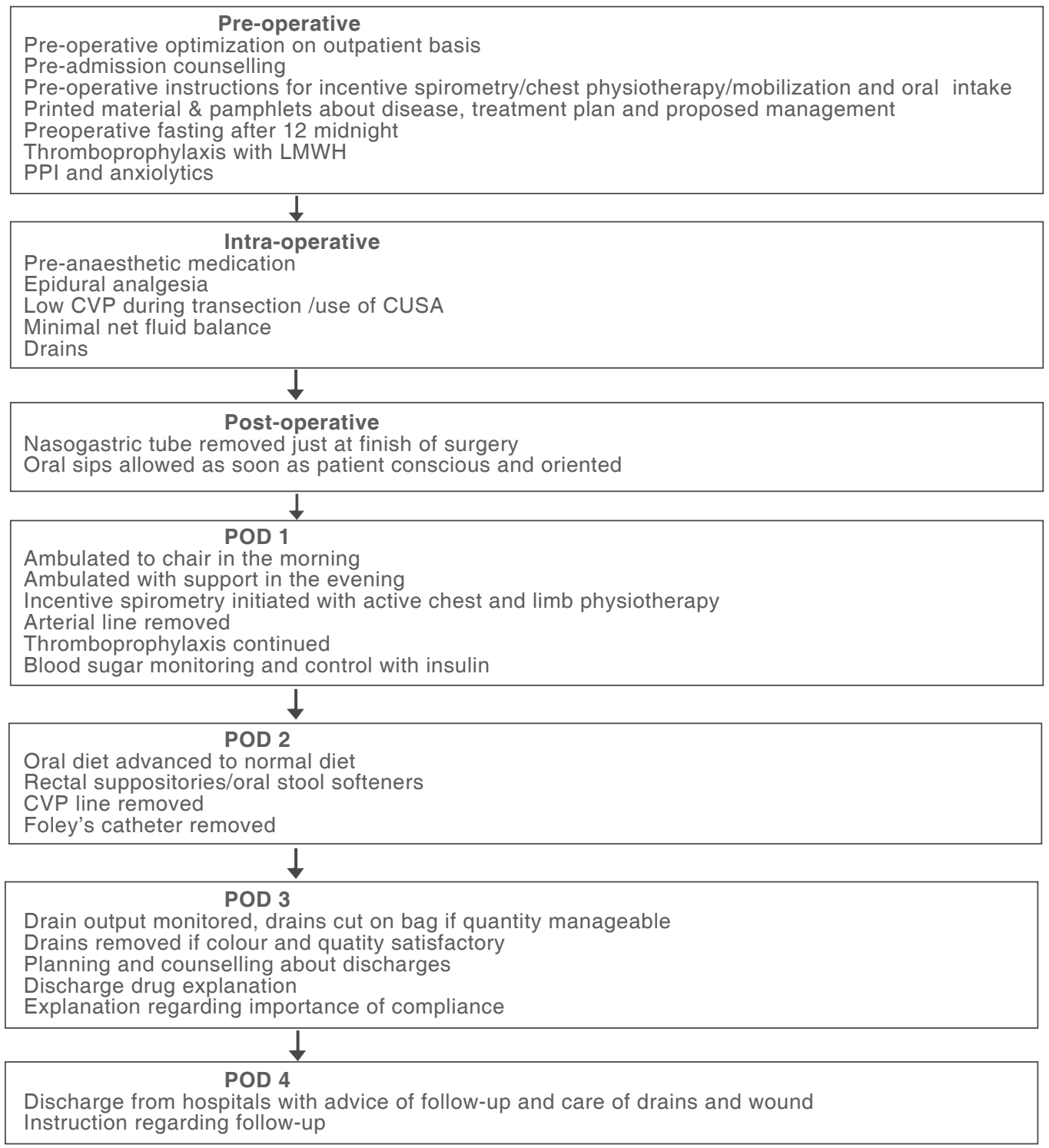

Figure 1. Summary of the enhanced recovery after surgery protocol applied at our institute. LMWH: low molecular weight heparin; PPI: proton-pump inhibitor; CVP: central venous pressure; CUSA: cavitron ultrasonic surgical aspirator; POD: postoperative day 


\section{CONCLUSION}

ERAS programs represent a standardized and evidence-based multimodal perioperative pathway founded on a series of measures aiming to attenuate the physical and psychological stress responses to surgical insults, potentiating postoperative rehabilitation of patients. Increasing evidence demonstrates that the application of ERAS in the field of liver surgery leads to an improvement in LOS, morbidity, patient satisfaction and a trend towards less hospital costs compared to traditional care. These benefits are leading to an increase adoption of various elements of ERAS protocols as part of modern surgical practice in liver surgery referral centers worldwide, including our institute [Figure 1]. Core elements of this multidisciplinary effort include adequate pre-operative patient education and counselling, shortened preoperative fasting with carbohydrate loading, judicious use of pre-anesthetic medication, prophylaxis against venous thromboembolism, targeted antimicrobial prophylaxis and early withdrawal, preventing and treating of PONV, minimally invasive approach, avoidance of postoperative nasogastric decompression, preventing hypothermia, optimal perioperative fluid management, selective use of abdominal drains, early urinary catheter removal, optimal pain control, early oral feeding and mobilization. Given the strong evidence suggesting that strict adherence to ERAS protocols is paramount for their successful implementation, continuous local audit of compliance has also become a key element of the approach ${ }^{[2,5]}$. Even though there is a growing body of evidence in favor of ERAS application in liver surgery, further studies are required to determine the most effective ERAS protocol for this particular field.

\section{DECLARATION}

\section{Authors' contributions}

Contributed significantly to the work, read the manuscript, attest to the validity and legitimacy of the data and its interpretation: Kumar $\mathrm{N}$

Contributed significantly to the work, read the manuscript, revised the manuscript, attest to the validity and legitimacy of the data and its interpretation: Jha SK

Conceptualised the review, contributed significantly to the work, read the manuscript, attest to the validity and legitimacy of the data and its interpretation: Negi SS

\section{Availability of data and materials}

Not applicable.

\section{Financial support and sponsorship}

None.

\section{Conflicts of interest}

All authors declared that there are no conflicts of interest.

\section{Ethical approval and consent to participate}

Not applicable.

\section{Consent for publication}

Not applicable.

\section{Copyright}

(c) The Author(s) 2018.

\section{REFERENCES}

1. Kehlet H. Manipulation of the metabolic response in clinical practice. World J Surg 2000;24:690-5.

2. Page AJ, Ejaz A, Spolverato G, Zavadsky T, Grant MC, et al. Enhanced recovery after surgery protocols for open hepatectomy--physiol- 
ogy, immunomodulation, and implementation. J Gastrointest Surg 2015;19:387-99.

3. Bardram L, Funch-Jensen P, Jensen P, Crawford ME, Kehlet H. Recovery after laparoscopic colonic surgery with epidural analgesia, and early oral nutrition and mobilisation. Lancet 1995;345:763-4.

4. Ljungqvist O, Scott M, Fearon KC. Enhanced recovery after surgery: a review. JAMA Surg 2017;152:292-8.

5. Melloul E, Hübner M, Scott M, Snowden C, Prentis J, et al. Guidelines for perioperative care for liver surgery: enhanced recovery after surgery (ERAS) society recommendations. World J Surg 2016;40:2425-40.

6. MacKay G, O’Dwyer PJ. Early discharge following liver resection for colorectal metastases. Scott Med J 2008;53:22-4.

7. van Dam RM, Hendry PO, Coolsen MM, Bemelmans MH, Lassen K, et al. Initial experience with a multimodal enhanced recovery programme in patients undergoing liver resection. Br J Surg 2008;95:969-75.

8. Lin DX, Li X, Ye QW, Lin F, Li LL, et al. Implementation of a fast-track clinical pathway decreases postoperative length of stay and hospital charges for liver resection. Cell Biochem Biophys 2011;61:413-9.

9. Wang C, Zheng G, Zhang W, Zhang F, Lv S, et al. Enhanced recovery after surgery programs for liver resection: a meta-analysis. J Gastrointest Surg 2017;21:472-86.

10. Wong-Lun-Hing EM, van Dam RM, Heijnen LA, Busch OR, Terkivatan T, et al. Is current perioperative practice in hepatic surgery based on enhanced recovery after surgery (ERAS) principles? World J Surg 2014;38:1127-40.

11. Gouin JP, Kiecolt-Glaser JK. The impact of psychological stress on wound healing: methods and mechanisms. Crit Care Nurs Clin North Am 2012;24:201-13.

12. Lassen K, Coolsen MM, Slim K, Carli F, de Aguilar-Nascimento JE, et al. Guidelines for perioperative care for pancreaticoduodenectomy: enhanced recovery after surgery (ERAS®) society recommendations. World J Surg 2013;37:240-58.

13. Disbrow EA, Bennett HL, Owings JT. Effect of preoperative suggestion on postoperative gastrointestinal motility. West J Med 1993;158:488-92.

14. Witte CL, Witte MH. Ileus and ignorance. West J Med 1993;158:532-4.

15. Smith I, Kranke P, Murat I, Smith A, O’Sullivan G, et al. Perioperative fasting in adults and children: guidelines from the European society of anaesthesiology. Eur J Anaesthesiol 2011;28:556-69.

16. Nygren J, Thorell A, Jacobsson H, Larsson S, Schnell PO, et al. Preoperative gastric emptying. Effects of anxiety and oral carbohydrate administration. Ann Surg 1995;222:728-34.

17. Sutherland AD, Stock JG, Davies JM. Effects of preoperative fasting on morbidity and gastric contents in patients undergoing day-stay surgery. Br J Anaesth 1986;58:876-8.

18. Agarwal A, Chari P, Singh H. Fluid deprivation before operation. The effect of a small drink. Anaesthesia 1989;44:632-4.

19. Brady M, Kinn S, Stuart P. Preoperative fasting for adults to prevent perioperative complications. Cochrane Database Syst Rev 2003;(4):CD004423.

20. Thorell A, Nygren J, Ljungqvist O. Insulin resistance: a marker of surgical stress. Curr Opin Clin Nutr Metab Care 1999;2:69-78.

21. Bilku DK, Dennison AR, Hall TC, Metcalfe MS, Garcea G. Role of preoperative carbohydrate loading: a systematic review. Ann R Coll Surg Engl 2014;96:15-22.

22. Hausel J, Nygren J, Thorell A, Lagerkranser M, Ljungqvist O. Randomized clinical trial of the effects of oral preoperative carbohydrates on postoperative nausea and vomiting after laparoscopic cholecystectomy. Br J Surg 2005;92:415-21.

23. Henriksen MG, Hessov I, Dela F, Hansen HV, Haraldsted V, et al. Effects of preoperative oral carbohydrates and peptides on postoperative endocrine response, mobilization, nutrition and muscle function in abdominal surgery. Acta Anaesthesiol Scand 2003;47:191-9.

24. Mathur S, Plank LD, McCall JL, Shapkov P, Mcllroy K, et al. Randomized controlled trial of preoperative oral carbohydrate treatment in major abdominal surgery. Br J Surg 2010;97:485-94.

25. Dimick JB, Pronovost PJ, Cowan JA, Lipsett PA. Complications and costs after high-risk surgery: where should we focus quality improvement initiatives? J Am Coll Surg 2003;196:671-8.

26. Uchiyama K, Ueno M, Ozawa S, Kiriyama S, Kawai M, et al. Risk factors for postoperative infectious complications after hepatectomy. J Hepatobiliary Pancreat Sci 2011;18:67-73.

27. Garwood RA, Sawyer RG, Thompson L, Adams RB. Infectious complications after hepatic resection. Am Surg 2004;70:787-92.

28. Sugawara G, Ebata T, Yokoyama Y, Igami T, Takahashi Y, et al. The effect of preoperative biliary drainage on infectious complications after hepatobiliary resection with cholangiojejunostomy. Surgery 2013;153:200-10.

29. Wu CC, Yeh DC, Lin MC, Liu TJ, P'eng FK. Prospective randomized trial of systemic antibiotics in patients undergoing liver resection. Br J Surg 1998;85:489-93.

30. Hirokawa F, Hayashi M, Miyamoto Y, Asakuma M, Shimizu T, et al. Evaluation of postoperative antibiotic prophylaxis after liver resection: a randomized controlled trial. Am J Surg 2013;206:8-15.

31. Togo S, Tanaka K, Matsuo K, Nagano Y, Ueda M, et al. Duration of antimicrobial prophylaxis in patients undergoing hepatectomy: a prospective randomized controlled trial using flomoxef. J Antimicrob Chemother 2007;59:964-70.

32. Sugawara G, Yokoyama Y, Ebata T, Mizuno T, Yagi T, et al. Duration of antimicrobial prophylaxis in patients undergoing major hepatectomy with extrahepatic bile duct resection: a randomized controlled trial. Ann Surg 2018;267:142-8.

33. Gurusamy KS, Naik P, Davidson BR. Methods of decreasing infection to improve outcomes after liver resections. Cochrane Database Syst Rev 2011; doi: 10.1002/14651858.CD006933.pub2.

34. De Pietri L, Montalti R, Begliomini B, Scaglioni G, Marconi G, et al. Thromboelastographic changes in liver and pancreatic cancer surgery: hypercoagulability, hypocoagulability or normocoagulability? Eur J Anaesthesiol 2010;27:608-16.

35. Dondero F, Taillé C, Mal H, Sommacale D, Sauvanet A, et al. Respiratory complications: a major concern after right hepatectomy in living liver donors. Transplantation 2006;81:181-6.

36. Bezeaud A, Denninger MH, Dondero F, Saada V, Venisse L, et al. Hypercoagulability after partial liver resection.Thromb Haemost 2007;98:1252-6.

37. Cerutti E, Stratta C, Romagnoli R, Schellino MM, Skurzak S, et al. Thromboelastogram monitoring in the perioperative period of hepatectomy for adult living liver donation. Liver Transpl 2004;10:289-94. 
38. Melloul E, Dondéro F, Vilgrain V, Raptis DA, Paugam-Burtz C, et al. Pulmonary embolism after elective liver resection: a prospective analysis of risk factors. J Hepatol 2012;57:1268-75.

39. Reddy SK, Turley RS, Barbas AS, Steel JL, Tsung A, et al. Post-operative pharmacologic thromboprophylaxis after major hepatectomy: does peripheral venous thromboembolism prevention outweigh bleeding risks? J Gastrointest Surg 2011;15:1602-10.

40. Rasmussen MS, Jørgensen LN, Wille-Jørgensen P. Prolonged thromboprophylaxis with low molecular weight heparin for abdominal or pelvic surgery. Cochrane Database Syst Rev 2009; doi: 10.1002/14651858.CD004318.pub2.

41. Mastoraki A, Mastoraki S, Schizas D, Patras R, Krinos N, et al. Facing the challenge of venous thromboembolism prevention in patients undergoing major abdominal surgical procedures for gastrointestinal cancer. World J Gastrointest Oncol 2018;10:328-35.

42. Reich H, McGlynn F, DeCaprio J, Budin R. Laparoscopic excision of benign liver lesions. Obstet Gynecol 1991;78:956-8.

43. Gagner M, Rheault M, Dubuc J. Laparoscopic partial hepatectomy for liver tumor. Surg Endosc 1992;6:97-8.

44. Azagra JS, Goergen M, Gilbart E, Jacobs D. Laparoscopic anatomical (hepatic) left lateral segmentectomy-technical aspects. Surg Endosc 1996;10:758-61.

45. Wakabayashi G, Cherqui D, Geller DA, Han HS, Kaneko H, et al. Laparoscopic hepatectomy is theoretically better than open hepatectomy: preparing for the 2nd International Consensus Conference on laparoscopic liver resection. J Hepatobiliary Pancreat Sci 2014;21:723-31.

46. Nguyen KT, Gamblin TC, Geller DA. World review of laparoscopic liver resection-2,804 patients. Ann Surg 2009;250:831-41.

47. Buell JF, Cherqui D, Geller DA, O'Rourke N, Iannitti D, et al. The international position on laparoscopic liver surgery: the Louisville statement, 2008. Ann Surg 2009;250:825-30.

48. Wakabayashi G, Cherqui D, Geller DA, Buell JF, Kaneko H, et al. Recommendations for laparoscopic liver resection: a report from the second international consensus conference held in Morioka. Ann Surg 2015;261:619-29.

49. Abu Hilal M, McPhail MJ, Zeidan B, Zeidan S, Hallam MJ, et al. Laparoscopic versus open left lateral hepatic sectionectomy: a comparative study. Eur J Surg Oncol 2008;34:1285-8.

50. Viganò L, Tayar C, Laurent A, Cherqui D. Laparoscopic liver resection: a systematic review. J Hepatobiliary Pancreat Surg 2009;16:410-21.

51. Giuliani A, Migliaccio C, Ceriello A, Aragiusto G, La Manna G, et al. Laparoscopic vs. open surgery for treating benign liver lesions: assessing quality of life in the first year after surgery. Updates Surg 2014;66:127-33.

52. Morise Z, Ciria R, Cherqui D, Chen KH, Belli G, et al. Can we expand the indications for laparoscopic liver resection? A systematic review and meta-analysis of laparoscopic liver resection for patients with hepatocellular carcinoma and chronic liver disease. J Hepatobiliary Pancreat Sci 2015;22:342-52.

53. Guro H, Cho JY, Han HS, Yoon YS, Choi Y, et al. Current status of laparoscopic liver resection for hepatocellular carcinoma. Clin Mol Hepatol 2016;22:212-8.

54. Fretland ÅA, Dagenborg VJ, Bjørnelv GMW, Kazaryan AM, Kristiansen R, et al. Laparoscopic versus open resection for colorectal liver metastases: the OSLO-COMET randomized controlled trial. Ann Surg 2018;267:199-207.

55. Liu Z, Ding H, Xiong X, Huang Y. Laparoscopic left lateral hepatic sectionectomy was expected to be the standard for the treatment of left hepatic lobe lesions: a meta-analysis. Medicine (Baltimore) 2018;97:e9835.

56. Stoot JH, van Dam RM, Busch OR, van Hillegersberg R, De Boer M, et al. The effect of a multimodal fast-track programme on outcomes in laparoscopic liver surgery: a multicentre pilot study. HPB (Oxford) 2009;11:140-4.

57. Ratti F, Cipriani F, Reineke R, Comotti L, Paganelli M, et al. The clinical and biological impacts of the implementation of fast-track perioperative programs in complex liver resections: a propensity score-based analysis between the open and laparoscopic approaches. Surgery 2018;164:395-403.

58. Wangensteen OH. Inhibition ileus (paralytic), In The Therapeutic Problem in Bowel Obstruction. Springfield, Ill, CC Thomas, 1937

59. Pessaux P, Regimbeau JM, Dondéro F, Plasse M, Mantz J, et al. Randomized clinical trial evaluating the need for routine nasogastric decompression after elective hepatic resection. Br J Surg 2007;94:297-303.

60. Ephgrave KS, Kleiman-Wexler R, Pfaller M, Booth B, Werkmeister L, et al. Postoperative pneumonia: a prospective study of risk factors and morbidity. Surgery 1993;114:815-9; discussion 819-21.

61. Dureuil B, Viirès N, Cantineau JP, Aubier M, Desmonts JM. Diaphragmatic contractility after upper abdominal surgery. J Appl Physiol (1985) 1986;61:1775-80.

62. Olesen KL, Birch M, Bardram L, Burcharth F. Value of nasogastric tube after colorectal surgery. Acta Chir Scand 1984;150:251-3.

63. Vermeulen H, Storm-Versloot MN, Busch OR, Ubbink DT. Nasogastric intubation after abdominal surgery: a meta-analysis of recent literature. Arch Surg 2006;141:307-14.

64. Bauer JJ, Gelernt IM, Salky BA, Kreel I. Is routine postoperative nasogastric decompression really necessary? Ann Surg 1985;201:2336.

65. Friedman M, Baim H, Shelton V, Stobnicki M, Chilis T, et al. Laryngeal injuries secondary to nasogastric tubes. Ann Otol Rhinol Laryngol 1981;90:469-74.

66. Manning BJ, Winter DC, McGreal G, Kirwan WO, Redmond HP. Nasogastric intubation causes gastroesophageal reflux in patients undergoing elective laparotomy. Surgery 2001;130:788-91.

67. Taylor FW. Electrolyte loss by postoperative nasal-gastric suction. AMA Arch Surg 1953;66:538-44.

68. Nelson R, Edwards S, Tse B. Prophylactic nasogastric decompression after abdominal surgery. Cochrane Database Syst Rev 2007;(3):CD004929.

69. Ichida H, Imamura H, Yoshimoto J, Sugo H, Ishizaki Y, et al. Randomized controlled trial for evaluation of the routine use of nasogastric tube decompression after elective liver surgery. J Gastrointest Surg 2016;20:1324-30.

70. Petrowsky H, Demartines N, Rousson V, Clavien PA. Evidence-based value of prophylactic drainage in gastrointestinal surgery: a systematic review and meta-analyses. Ann Surg 2004;240:1074-84; discussion 1084-5.

71. Wada S, Hatano E, Yoh T, Seo S, Taura K, et al. Is routine abdominal drainage necessary after liver resection? Surg Today 2017;47:712-7.

72. Gurusamy KS, Samraj K, Davidson BR. Routine abdominal drainage for uncomplicated liver resection. Cochrane Database Syst Rev 
2007;(3):CD006232.

73. Squires MH 3rd, Lad NL, Fisher SB, Kooby DA, Weber SM, et al. Value of primary operative drain placement after major hepatectomy: a multi-institutional analysis of 1,041 patients. J Am Coll Surg 2015;220:396-402.

74. Sun HC, Qin LX, Lu L, Wang L, Ye QH, et al. Randomized clinical trial of the effects of abdominal drainage after elective hepatectomy using the crushing clamp method. Br J Surg 2006;93:422-6.

75. Kim YI, Fujita S, Hwang VJ, Nagase Y. Comparison of abdominal drainage and no-drainage after elective hepatectomy: a randomized study. Hepatogastroenterology 2014;61:707-11.

76. Brower RG. Consequences of bed rest. Crit Care Med 2009;37:S422-8

77. Yip VS, Dunne DF, Samuels S, Tan CY, Lacasia C, et al. Adherence to early mobilisation: key for successful enhanced recovery after liver resection. Eur J Surg Oncol 2016;42:1561-7.

78. Zaouter C, Kaneva P, Carli F. Less urinary tract infection by earlier removal of bladder catheter in surgical patients receiving thoracic epidural analgesia. Reg Anesth Pain Med 2009;34:542-8.

79. Ni CY, Wang ZH, Huang ZP, Zhou H, Fu LJ, et al. Early enforced mobilization after liver resection: a prospective randomized controlled trial. Int J Surg 2018;54:254-8.

80. Lassen K, Kjaeve J, Fetveit T, Tranø G, Sigurdsson HK, et al. Allowing normal food at will after major upper gastrointestinal surgery does not increase morbidity: a randomized multicenter trial. Ann Surg 2008;247:721-9.

81. Hendry PO, van Dam RM, Bukkems SF, McKeown DW, Parks RW, et al. Randomized clinical trial of laxatives and oral nutritional supplements within an enhanced recovery after surgery protocol following liver resection. Br J Surg 2010;97:1198-206.

82. Maeda H, Okabayashi T, Nishimori I, Yamashita K, Sugimoto T, et al. Hyperglycemia during hepatic resection: continuous monitoring of blood glucose concentration. Am J Surg 2010;199:8-13.

83. Hanazaki K, Munekage M, Ichikawa K, Okabayashi T. Ischemic preconditioning may reduce hyperglycemia associated with intermittent Pringle maneuver in hepatic resection. J Hepatobiliary Pancreat Sci 2013;20:257.

84. Durczynski A, Strzelczyk J, Wojciechowska-Durczynska K, Borkowska A, Hogendorf P, et al. Major liver resection results in early exacerbation of insulin resistance, and may be a risk factor of developing overt diabetes in the future. Surg Today 2013;43:534-8.

85. Carlisle JB, Stevenson CA. Drugs for preventing postoperative nausea and vomiting. Cochrane Database Syst Rev 2006;(3):CD004125.

86. Henzi I, Walder B, Tramèr MR. Metoclopramide in the prevention of postoperative nausea and vomiting: a quantitative systematic review of randomized, placebo-controlled studies. Br J Anaesth 1999;83:761-71.

87. Gan TJ, Diemunsch P, Habib AS, Kovac A, Kranke P, et al. Consensus guidelines for the management of postoperative nausea and vomiting. Anesth Analg 2014;118:85-113.

88. Saidi RF, Chang J, Verb S, Brooks S, Nalbantoglu I, Adsay V, Jacobs MJ. The effect of methylprednisolone on warm ischemia-reperfusion injury in the liver. Am J Surg 2007;193:345-7; discussion 347-8.

89. Alegre ML, Vandenabeele P, Depierreux M, Florquin S, Deschodt-Lanckman M, et al. Cytokine release syndrome induced by the 1452C11 anti-CD3 monoclonal antibody in mice: prevention by high doses of methylprednisolone. J Immunol 1991;146:1184-91.

90. Richardson AJ, Laurence JM, Lam VW. Use of pre-operative steroids in liver resection: a systematic review and meta-analysis. HPB (Oxford) 2014;16:12-9.

91. Li N, Gu WL, Weng JF, Lin F, Zhu GH, et al. Short-term administration of steroids does not affect postoperative complications following liver resection: evidence from a meta-analysis of randomized controlled trials. Hepatol Res 2015;45:201-9.

92. Schmidt SC, Hamann S, Langrehr JM, Höflich C, Mittler J, et al. Preoperative high-dose steroid administration attenuates the surgical stress response following liver resection: results of a prospective randomized study. J Hepatobiliary Pancreat Surg 2007;14:484-92.

93. Yamashita Y, Shimada M, Hamatsu T, Rikimaru T, Tanaka S, et al. Effects of preoperative steroid administration on surgical stress in hepatic resection: prospective randomized trial. Arch Surg 2001;136:328-33.

94. Streetz KL, Luedde T, Manns MP, Trautwein C. Interleukin 6 and liver regeneration. Gut 2000;47:309-12.

95. Glanemann M, Strenziok R, Kuntze R, Münchow S, Dikopoulos N, et al. Ischemic preconditioning and methylprednisolone both equally reduce hepatic ischemia/reperfusion injury. Surgery 2004;135:203-14.

96. Spelt L, Ansari D, Sturesson C, Tingstedt B, Andersson R. Fast-track programmes for hepatopancreatic resections: where do we stand? HPB (Oxford) 2011;13:833-8.

97. Jones C, Kelliher L, Dickinson M, Riga A, Worthington T, et al. Randomized clinical trial on enhanced recovery versus standard care following open liver resection. Br J Surg 2013;100:1015-24.

98. Ni CY, Yang Y, Chang YQ, Cai H, Xu B, et al. Fast-track surgery improves postoperative recovery in patients undergoing partial hepatectomy for primary liver cancer: a prospective randomized controlled trial. Eur J Surg Oncol 2013;39:542-7.

99. Lei Q, Wang X, Tan S, Xia X, Zheng H, et al. Fast-track programs versus traditional care in hepatectomy: a meta-analysis of randomized controlled trials. Dig Surg 2014;31:392-9.

100. Ni TG, Yang HT, Zhang H, Meng HP, Li B. Enhanced recovery after surgery programs in patients undergoing hepatectomy: a metaanalysis. World J Gastroenterol 2015;21:9209-16.

101. Li M, Zhang W, Jiang L, Yang J, Yan L. Fast track for open hepatectomy: a systemic review and meta-analysis. Int J Surg 2016;36:81-9.

102. Rouxel P, Beloeil H. Enhanced recovery after hepatectomy: a systematic review. Anaesth Crit Care Pain Med 2018; doi: 10.1016/ j.accpm.2018.05.003.

103. He F, Lin X, Xie F, Huang Y, Yuan R. The effect of enhanced recovery program for patients undergoing partial laparoscopic hepatectomy of liver cancer. Clin Transl Oncol 2015;17:694-701.

104. Joliat GR, Labgaa I, Hübner M, Blanc C, Griesser AC, et al. Cost-benefit analysis of the implementation of an enhanced recovery program in liver surgery. World J Surg 2016;40:2441-50.

105. Liang X, Ying H, Wang H, Xu H, Yu H, et al. Enhanced recovery program versus traditional care in laparoscopic hepatectomy. Medicine (Baltimore) 2016;95:e2835. 